\title{
BMJ Global Health Interventions to promote access to eyecare for non-dominant ethnic groups in high-income countries: a scoping review
}

\author{
Lisa M Hamm (D) , ${ }^{1}$ Aryati Yashadhana (D) , ${ }^{2,3,4,5}$ Helen Burn (D) , Joanna Black (D) , \\ Corina Grey (D) , ${ }^{7,8}$ Matire Harwood (D) , ${ }^{7}$ Roshini Peiris-John (D) , 7 \\ Matthew J Burton (D) , ${ }^{6,9}$ Jennifer R Evans (D) , Jacqueline Ramke (D) ${ }^{1,6}$
}

To cite: Hamm LM, Yashadhana A, Burn $\mathrm{H}$, et al. Interventions to promote access to eyecare for nondominant ethnic groups in highincome countries: a scoping review. BMJ Global Health 2021;6:e006188. doi:10.1136/ bmjgh-2021-006188

Handling editor Seye Abimbola

- Additional supplemental material is published online only. To view, please visit the journal online (http://dx.doi.org/10. 1136/bmjgh-2021-006188)

Received 9 May 2021 Accepted 11 August 2021

Check for updates

(c) Author(s) (or their employer(s)) 2021. Re-use permitted under CC BY. Published by BMJ.

For numbered affiliations see end of article.

Correspondence to

Dr Lisa M Hamm;

I.hamm@auckland.ac.nz

\section{ABSTRACT}

Purpose People who are distinct from the dominant ethnic group within a country can experience a variety of barriers to accessing eyecare services. We conducted a scoping review to map published interventions aimed at improving access to eyecare for non-Indigenous, non-dominant ethnic groups residing in high-income countries.

Methods We searched MEDLINE, Embase and Global Health for studies that described an intervention to promote access to eyecare for the target population. Two authors independently screened titles and abstracts followed by review of the full text of potentially relevant sources. For included studies, data extraction was carried out independently by two authors. Findings were summarised using a combination of descriptive statistics and thematic analysis.

Results We screened 5220 titles/abstracts, of which 82 reports describing 67 studies met the inclusion criteria. Most studies were conducted in the USA (90\%), attempted to improve access for Black (48\%) or Latinx (28\%) communities at-risk for diabetic retinopathy (42\%) and glaucoma (18\%). Only $30 \%$ included the target population in the design of the intervention; those that did tended to be larger, collaborative initiatives, which addressed both patient and provider components of access. Forty-eight studies $(72 \%)$ evaluated whether an intervention changed an outcome measure. Among these, attendance at a follow-up eye examination after screening was the most common ( $n=20 / 48,42 \%)$, and directly supporting patients to overcome barriers to attendance was reported as the most effective approach. Building relationships between patients and providers, running coordinated, longitudinal initiatives and supporting reduction of root causes for inequity (education and economic) were key themes highlighted for success.

Conclusion Although research evaluating interventions for non-dominant, non-Indigenous ethnic groups exist, key gaps remain. In particular, the paucity of relevant studies outside the USA needs to be addressed, and target communities need to be involved in the design and implementation of interventions more frequently.

\section{Key questions}

What is already known?

- Non-dominant ethnic groups living in high-income countries face worse eye health and vision outcomes compared to dominant ethnic groups.

- In high-income countries, most differences in eye health and vision outcomes between population groups are due to inequity.

What are the new findings?

- To date, most research about interventions to overcome inequitable access to eyecare for nondominant ethnic groups has been conducted in the USA, targeting Black and Latinx Americans.

- Only about one-third of the studies reported being designed in collaboration with the target population; papers which were interconnected with wider research groups were more likely to work with communities than smaller research initiatives.

- Approaches which promoted at-risk patients to attend follow-up appointments after community screening have been subject to the most evaluation.

What do the new findings imply?

- The geographical scope of the research relevant to this topic is very limited; high-income countries outside the USA should investigate and address inequity across ethnic groups in access to eyecare.

- New initiatives implementing strategies to improve access to eyecare for non-dominant ethnic groups should start with genuine relationships (including collaborative design), have a plan for longitudinal involvement, and support empowerment of the target community beyond eyecare.

\section{INTRODUCTION}

\section{Rationale}

The United Nations' Sustainable Development Goals aim to 'leave no one behind', however, many societal structures exist which systematically marginalise some groups while benefiting others. ${ }^{2}$ Specifically, allocation of 
resource tends to align with the interests, values, and norms of the 'dominant' group, being those with the highest concentration of wealth and decision-making power. ${ }^{2}$ In the context of Western high-income countries, dominant groups tend to align with the sociocultural norms and values of whiteness and neoliberalism. The establishment and maintenance of the socioeconomic gap between dominant and non-dominant ethnic groups is a reflection of structural racism. ${ }^{2-4}$ Here, we consider 'race' as a social construct which uses visual phenotyping and ancestry to justify systems of oppression and privilege, and 'structural racism' as the ways in which these systems of oppression and privilege (including education, criminal justice and health) are fostered. ${ }^{4}$ Pervasive structural racism in high-income countries means that non-dominant ethnic groups are often left behind, ${ }^{5}$ as evidenced most recently by mortality due to COVID-19 across the USA. ${ }^{3}$

Indigenous peoples worldwide have endured profound losses through colonisation and structural racism, as have many other non-dominant ethnic groups including Black, ${ }^{35}$ Latinx, Asian and Pacific Peoples. ${ }^{4}$ Since we report on Indigenous groups in a parallel report, ${ }^{6}$ here we focus on non-Indigenous, non-dominant ethnic groups (henceforth referred to as 'non-dominant'). Health inequities exist on several fronts, ${ }^{78}$ including decreased access to preventative medicine, ${ }^{9}$ higher burden of disease ${ }^{3}$ and poorer access to assessment and treatment ${ }^{10}$ as well as palliative care. ${ }^{11}$ Similar trends are apparent within eyecare. ${ }^{12-15}$ Black and Latinx people in the USA are less likely than white people to report having visited an eye care provider. ${ }^{16}$ Seasonal migrant workers (typically from non-dominant ethnicities) spend long hours exposed to irritants which can cause infection, irritation or injury to the eyes, ${ }^{17}$ while having limited access to eyecare for resulting issues. Children from non-dominant ethnic groups are more likely to have unmet eyecare needs. ${ }^{18}$

Barriers to accessing eyecare are extensive, ranging from knowledge about eye conditions, ${ }^{19}$ capacity to cover primary and secondary costs, and a lack of trust in the institutions providing care. Although complex, barriers can be conceptualised through theoretical frameworks of access, such as that proposed by Levesque $e t a l .^{20}$ This framework suggests that for a patient to move from identifying a healthcare need to having that need fulfilled, five steps are necessary, each of which requires medical providers to be accessible, and a patient to have the capacity to participate. From the provider perspective, Levesque $e t a t^{20}$ argue that this includes: (1) approachability, (2) acceptability, (3) availability, (4) affordability and (5) appropriateness. On the demand side, patients need to be supported in their abilities to: (1) perceive, (2) seek, (3) reach, (4) pay and (5) engage. These categories are interdependent; for example, a noted barrier for non-dominant ethnic groups is a fear that seeking healthcare might compromise immigration status, ${ }^{21}$ this may reflect a decreased capacity to seek, and potentially poor provider approachability and acceptability. Similarly, communication barriers, where the provider does not share a patient's language or cultural heritage ${ }^{22} 23$ could be conceptualised as an issue with acceptability, appropriateness or ability to engage. Notwithstanding the complexities, the framework is useful to identify barriers and consider ways to mitigate them.

\section{Aims}

In this report, we sought to summarise published literature on interventions to promote access to eyecare for non-Indigenous, non-dominant ethnic groups living in high-income countries. The overarching aim was broken down into three questions:

1. What is the extent of the published literature?

2. What can we learn from reported effectiveness of interventions?

3. What can we learn from authors' reflections on the potential to improve on interventions?

Similar questions have been raised by other researchers, but previous reviews have focused on improving access to eyecare in either low-income to middle-income countries ${ }^{2425}$ or high-income countries, ${ }^{26}{ }^{27}$ rather than exploring ethnicity-based inequities within a country. The goal of this, and our parallel paper, ${ }^{6}$ is to fill this gap for high-income countries.

\section{METHODS \\ Protocol and registration}

The study protocol was published ${ }^{28}$; the protocol and this study are reported according to the Preferred Reporting Items for Systematic Reviews and Meta-Analyses extension for Scoping Reviews guideline. ${ }^{29}$

\section{Patient and public involvement}

It was not feasible to include patient or public engagement in this review.

\section{Definitions and eligibility criteria}

- Population: As outlined in our protocol, ${ }^{28}$ the target population is difficult to define. Self-identification of ethnicity is often fluid and nuanced, ${ }^{30}$ and appropriate terminology is actively debated. ${ }^{31}$ We opted for the term 'non-dominant', aligned with Benson's definition that 'non-dominant groups are minorities or even majorities that are unfairly marginalised in certain social contexts' ${ }^{32}$ Since we addressed eyecare for Indigenous populations in a separate review, ${ }^{633}$ our target population for this review was limited to people who are not indigenous to the country where the study was conducted. We included studies in which at least $50 \%$ of participants were from a nondominant, non-indigenous ethnic group.

- Intervention: We sought studies that described interventions to improve access to eyecare, according to the Levesque framework. ${ }^{20}$ We only included general health studies (eg, about diabetes) if there was sufficient data on eyecare to be a stand-alone resource. 
- Setting: Studies needed to be conducted in highincome countries (as defined by the World Bank in 2019). ${ }^{34}$

- Study design: We included all studies which reported the impact of an intervention on participants, regardless of design, without a requirement for an evaluation of the intervention. Reviews, commentaries, editorials, and conference abstracts were excluded.

- Comparator: Studies with or without a comparator group were included. Studies with a comparator were considered 'evaluated'.

- Other: We included all languages and publication dates.

\section{Information sources}

An information specialist searched MEDLINE, Embase and Global Health databases as described ${ }^{28}$ on 28 July 2019 and updated on 26 August 2020. A sample database search is included online supplemental file 1, table S1.1.

\section{Selection of sources of evidence}

All results from the search were entered into Covidence (www.covidence.org) for screening. Two authors (from $\mathrm{LMH}, \mathrm{JR}, \mathrm{JB}, \mathrm{CG}$ and $\mathrm{HB}$ ) independently reviewed each title and abstract to exclude those that did not meet inclusion criteria. Disagreements were resolved between the two reviewers, with a third author consulted when needed. The full text of each selected article was reviewed independently by two authors (from LMH, AY, HB, JR and JB), to determine whether they should be included in the data extraction phase. Again, conflict resolution was handled by discussion, and a third reviewer was asked to consult when needed.

\section{Data charting process}

A data extraction form was developed based on the categories defined in our protocol ${ }^{24}$ and piloted by LMH, HB and JR. Simplifications which facilitated consistent data extraction are described below. Some proposed data extraction fields were eliminated after pilot extraction due to paucity, or inconsistent reporting of information (eg, socioeconomic status of participants). Data extraction was carried out independently for each publication by two authors (from LMH, RP-J, AY, JR, HB and JB).

\section{Data items}

What is the extent of the published literature?

Characteristics of the publication and targeted population

- Title, year, country.

- Targeted condition.

- Targeted ethnicity.

- Age: categorised as adult, child or all ages.

Characteristics of the intervention

- A free-text description of the intervention.

- Dimensions of Levesque framework addressed; this was recorded in ten binary entries, allowing the five dimensions of access to be assessed according to the patient and provider side independently (each included study contained between one and ten dimensions).

- Engagement with target population during development and implementation of the intervention (in most cases the study required a clear statement about community involvement, however, some publications were part of larger initiatives, so engagement with the target community was traced back through previous publications).

\section{What can we learn from reported effectiveness of interventions?}

- Study design: categorised according to types of comparator: none, sequential (pre-post) or concurrent (including alternate intervention or control).

- How many people participated.

- Free-text description of outcome measure.

- Whether the authors determined the intervention to be effective (this did not include an analysis of methodological quality).

\section{What can we learn from authors' reflections on the potential to improve on interventions?}

- Free text describing authors' reflections on strengths, weaknesses and recommendations.

\section{Data synthesis strategy}

\section{What is the extent of the published literature?}

We conducted a descriptive analysis of characteristics of studies and targeted groups, as well as of the characteristics of intervention strategies. Regarding targeted groups, if $>50 \%$ of the participants were reported from one ethnic group, we reported that group as the target population. Since the terms used to refer to ethnicity varied across reports (eg, Hispanic, Latino) we assigned consistent names to each group which emerged during data extraction. Group names (such as 'Black' and 'Latinx') were based on our current understanding of best practice for promoting inclusivity and respect, however, we recognise the drawbacks of their use; these terms reflect norms from specific contexts at this time, and do not capture the diversity of cultures and people within each category. Although most studies specifically targeted one ethnic group, some studies targeted a community-based factor other than ethnicity (eg, recent immigrants from varied backgrounds). If our inclusion criteria were still met ( $>50 \%$ non-dominant, non-Indigenous participants), but no single ethnic group targeted (eg, if the breakdown of participants was 25\% Pacific Peoples, 25\% Latinx and $25 \%$ Black) we used the term 'mixed ethnicities', to reflect the mixture of ethnic backgrounds targeted. Regarding interventions, we summed binary data from the Levesque framework section to categorise interventions as patient focused, provider focused or both. We also plotted author collaborations to understand how studies were linked within collaborative networks.

What can we learn from reported effectiveness of interventions? We limited our analysis in this section to studies with a comparator, and grouped them by outcome measure. If a 
study reported multiple outcomes across several papers, we reported each. However, if several outcomes were reported in a single paper, we used the outcome closest to behaviour (eg, attendance at a follow-up eye examination was recorded rather than knowledge about target condition, for cases in which both these outcomes were reported). We simplified descriptive text about outcome measures into the following categories:

- Survey (results from an assessment of health knowledge or perspectives about accessing eyecare).

- Attendance at screening.

- Attendance at follow-up eye exam (percentage or referred participants who attended a follow-up eye exam after screening-including self-reported and confirmed attendance, and all time frames).

- Adherence to (non-attendence) recommendations (including treatment and prevention measures).

- Health (eg, incidence of blindness, vision impairment, control of diabetes, etc).

We did not assess the quality of evidence, rather, we relied on the authors notes about the comparator, outcome measure and results, and categorised effectiveness as follows:

- Effective (improvement in outcome measured with statistical significance).

- Inconclusive (improvement in outcome measured but not reaching statistical significance, or a negative result but authors note a design flaw may have masked an important finding).

- Ineffective (no change in outcome measure, or a change in the opposite direction).

\section{What can we learn from authors' reflections on the potential to improve on interventions?}

To address the third question, a thematic analysis was conducted across all free-text (qualitative) data fields including authors' reflections on strengths, weaknesses and recommendations within each identified paper, to converge on perceived elements associated with 'success'. We used an inductive approach to coding, ${ }^{35}$ where iterative use of four phases of analysis (data familiarisation, generating initial codes, categorisation and emergence of themes, and reviewing of identified themes) was applied to qualatative data. A single author completed the analysis manually by categorising free-text data fields using an Excel spreadsheet and colour coding techniques to allow for thematic synthesis of data. We were interested in understanding the 'weight' of each theme across the included studies, and therefore, also captured frequency (eg, the number of papers that referred to an identified theme). The themes that were most referenced across the set of papers as a whole, are presented in the results section.

\section{Grey literature}

Although not planned, ${ }^{28}$ in response to review we checked whether additional initiatives were highlighted in unpublished literature (details of our grey literature

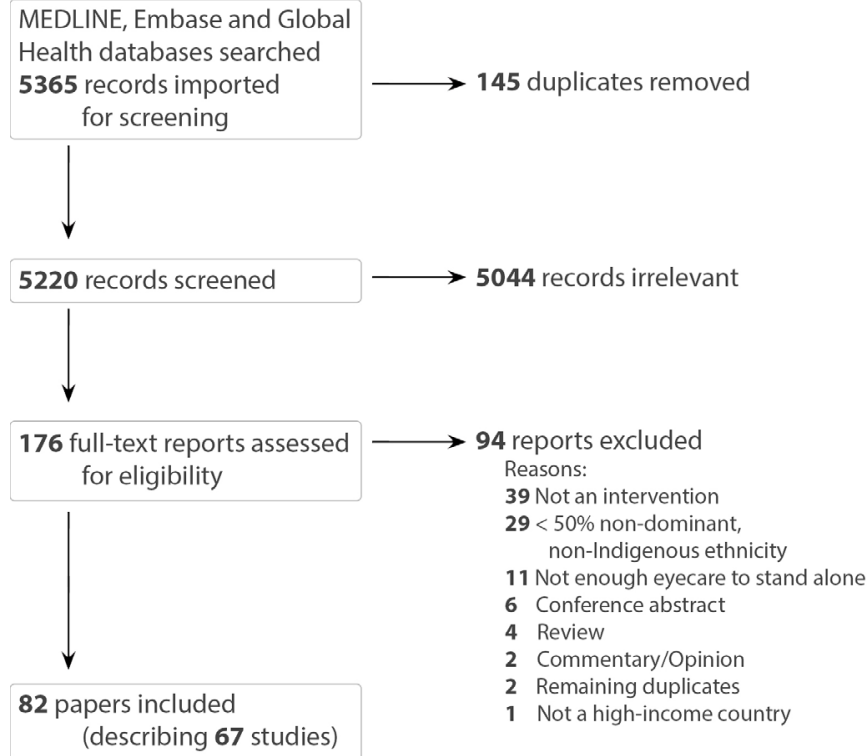

Figure 1 PRISMA flow diagram. PRISMA, Preferred Reporting Items for Systematic Reviews and Meta-Analyses.

search are available in online supplemental file 1, table S1.2). Unpublished initiatives surfacing from this additional search are summarised at the end of our results section, to provide additional context for our aims.

\section{RESULTS \\ Summary of sources of evidence}

Our search of the published literature identified 5220 unique records which were screened by title and abstract, and 176 were included in the full text screening. Eligible references included 82 papers, describing 67 studies (figure 1).

\section{What is the extent of the published literature?}

Characteristics of publication and targeted groups

Characteristics of publications and targeted groups are summarised in figure 2. The majority of research $(n=60$, $90 \%$ of studies, or $n=75,92 \%$ of papers) was conducted in the USA, targeting Black or Latinx Americans. Two studies from the $\mathrm{UK}^{1936}$ and one from Canada ${ }^{37}$ sought to improve eyecare for Asian populations. Research in The Netherlands, ${ }^{38}{ }^{39}$ Israel $^{40}$ and Australia ${ }^{41}$ did not focus on a specific ethnic group, rather each study aimed to improve access for non-dominant ethnicities more generally. Studies focused on adults targeted diabetic retinopathy $(n=28,42 \%)$, glaucoma $(n=12,18 \%)$ and injury prevention for seasonal farm workers $(n=7,10 \%)$. Twenty studies promoted access for general eye health, some for adults or all ages $(n=10,15 \%)$, and some specifically for children $(n=10,15 \%)$. Most papers were published in the last 20 years $(n=60,90 \%)$, with a spike in last 10 years $(n=40,60 \%)$, driven by work done in the USA.

\section{Characteristics of the interventions}

Figure 3 summarises the characteristics of the interventions. Most studies $(n=59,88 \%)$ addressed more than 

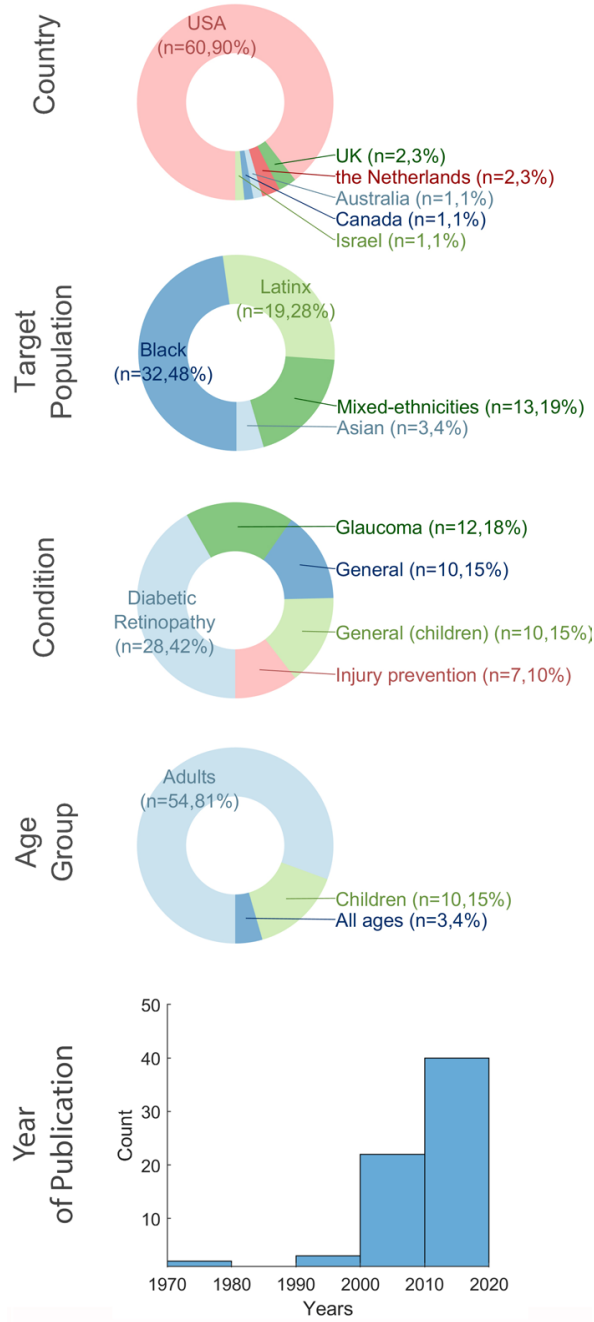

Figure 2 Characteristics of publications (studies) and target groups.

one dimension of access, with most emphasis on making services more approachable $(n=51,76 \%$, eg, running screening programs in convenient locations), and least emphasis on making eyecare more acceptable $(n=17$, $25 \%$, eg, training culturally sensitive clinical staff) ${ }^{42}$ and improving the patient's ability to pay $(\mathrm{n}=15,22 \% \mathrm{eg}$, providing financial incentives). ${ }^{43}$

Studies were relatively evenly split between focusing on the patient side of access $(n=28,42 \%)$, provider side $(n=21,31 \%)$ and integrating both $(n=18,27 \%)$. Approaches which focused on the provider side of access mostly described community screening $(\mathrm{n}=15,71 \%)$ and comprehensive community eyecare services $(n=3,14 \%)$, whereas approaches focused on the patient side of access described health education $(n=16,55 \%)$, and supporting people to access services $(n=13,45 \%$, including reminder calls and one-on-one counselling to identify and overcome barriers to access). Only 20 studies (30\%) reported having the target community involved in the design and implementation. Studies that involved the target community in the design were more likely to address both the patient and provider sides of access. Very few studies which engaged with the community ran isolated screening or service programmes.

For each paper, we recorded the number of other included papers connected through coauthorship, as well as the strength of the connection between papers (sum of common authors). Papers which involved the target community in the study design had more connections to other papers, and stronger connections between papers than those that did not (both $\mathrm{p}<0.001$ ). In other words, studies that involved the target community in the design were also more likely to originate within wide collaborative networks than more isolated initiatives. Figure 4 allows visualisation of collaborative networks by plotting authors by their connections to papers and targeted conditions (those which engaged with the target community in the design are highlighted in green).

\section{What can we learn from reported effectiveness of interventions?}

Most studies ( $\mathrm{n}=48,72 \%)$ included a comparator; of these, $20(42 \%)$ measured an outcome before and after an intervention, while $28(58 \%)$ compared alternative interventions concurrently. A summary of interventions, grouped by the comparator type, can be found in the online supplemental table S1.2. In this section, we summarise studies with a comparator, organised by outcome measure (surveys: 9/48, 19\%, screening: $5 / 48$, $10 \%$, eye exam: $20 / 48,42 \%$, non-attendance adherence: $8 / 48,17 \%$, health: $6 / 48,13 \%$ ).

\section{Survey of knowledge}

Implementation of a variety of educational programmes, including glaucoma ${ }^{44}$ diabetic retinopathy ${ }^{45-47}$ and general eye health for adults ${ }^{44}$ and children ${ }^{49}$ all resulted in improvements in knowledge (although not all statistically so), as did writing out instructions for glaucoma medications. ${ }^{50}$ Although these interventions improved knowledge, most authors highlighted the gap between self-reported knowledge, and a change in behaviour or action. ${ }^{19} 464851$

\section{Improving attendance at screening}

Five studies specifically quantified whether attendance improved as a result of an intervention, all reported improvements in screening rates after initiatives were launched..$^{41}{ }^{52-55}$ Recruitment initiatives ${ }^{41}$ and automated reminder calls ${ }^{56}$ were both highlighted as effective strategies to improve attendance.

\section{Improving attendance at follow-up eye exams}

Compared with measuring knowledge and attendance at screening appointments, studies which measured attendance at a follow-up eye exam were more likely to be inconclusive $(n=6 / 19,32 \%)$ or ineffective $(n=2 / 19,11 \%)$. Specifically, education programmes, although helpful to improve knowledge on surveys, showed limited success for improving attendance at follow-up eye exams, ${ }^{57-59}$ and having patients sign a contract stating that they will attend an appointment was ineffective. ${ }^{60}$ 
Dimensions of Levesque Framework addresed

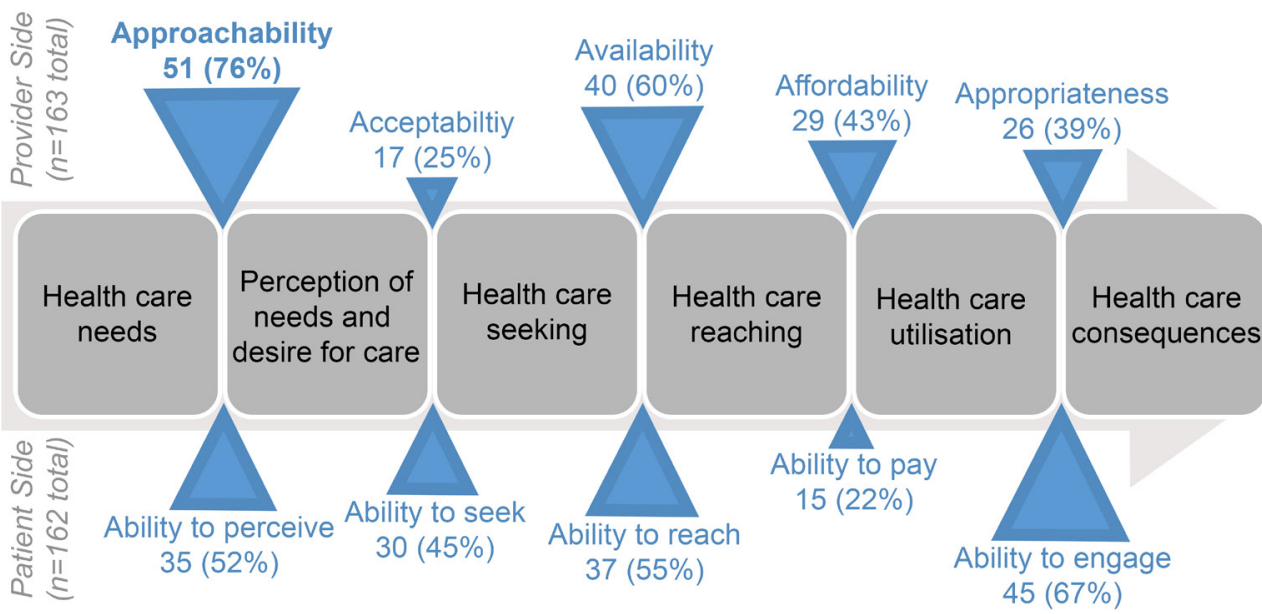

All that apply; each study could potentially address between 1 and 10 dimensions of access
Target community involved in study design
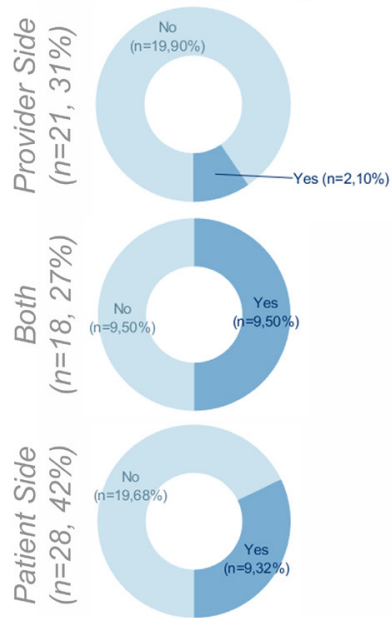

Best fit for each study

Figure 3 Characteristics of interventions, in terms of dimensions of access addressed and involvement of target community in study design. For the Levesque framework, a single study typically addressed more than one dimension of access (the denominator for each is the total number of studies). When studies were categorised as mainly addressing patient, provider or both sides of access, those which targeted both were most likely to have engaged with the target community.

Studies implementing supportive reminder calls were more frequently considered effective ${ }^{61-63}$ than improving education or having patients sign contracts, however, not universally so. For example, Meng $e t a l^{64}$ used reminder calls in a wider context (in an attempt to reduce ethnic disparities in Medicaid use) and found it was not effective for most groups. The nature of the supportive interaction appeared more important than whether or not the interaction took place. ${ }^{63}{ }^{65-67}$ For example, 'behavioural activation' (which includes a trained support person

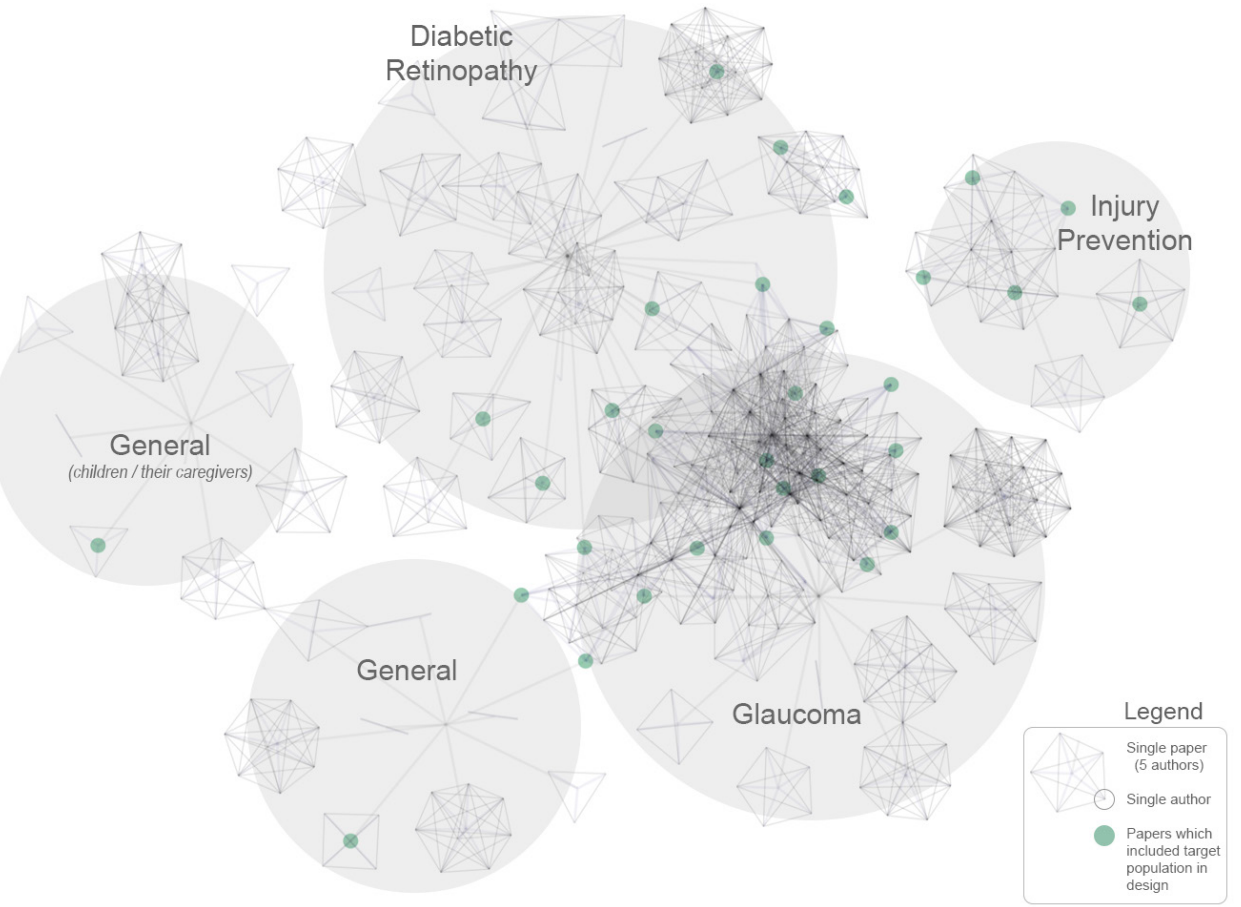

Figure 4 Summary of included publications by relationship between authors and conditions. Each node represents an author, and each cluster of nodes represents a paper, or groups of papers by similar authors. Papers which included the target population in the design are highlighted in green. Note the clustering of green dots within collaborative networks (although with notable exceptions). 
helping a patient to identify, and working through, barriers) was an effective approach in several included studies. ${ }^{65}$ 68-71 A study about Black Americans at risk of diabetic retinopathy ${ }^{65}$ (described across three papers ${ }^{65-67}$ showed behavioural activation to be more effective than more general supportive therapy (a placebo treatment where participants are provided one-on-one empathetic discussion about the impact of diabetes on their lives, in a comfortable environment). 'Patient navigators' likely fill a similar role to those who facilitate behavioural activation; they help guide patients through the steps of eyecare, providing support as needed. An initial randomised controlled trial (RCT) evaluating effectiveness of adding patient navigators within an already supportive eyecare programme showed inconclusive results ${ }^{72}$ but a follow-up RCT is underway. ${ }^{73-75}$ Hiring school nurses to help students navigate from screening to full eye exams and comply with treatment was reported to be effective compared with screening alone ${ }^{76}$ (although some universal childhood screening programmes were very effective at promoting follow-up). ${ }^{7}$

Initiatives with wider, integrated approaches also demonstrated effectiveness at improving attendance at follow-up eye exams (though not always significantly so). These initiatives tended to include several components, such as prescheduling follow-up appointments, ${ }^{43} 78$ running follow-up appointments at convenient locations, ${ }^{79}$ incorporating education elements ${ }^{43980}$ and providing financial incentives. ${ }^{4380}$

\section{Improving adherence with preventative measures or treatment}

Several studies showed that providing appropriate, free eyewear helps to promote use of protective eyewear among seasonal farm workers, ${ }^{81}$ though training respected peer-workers to provide education about eye health, ${ }^{1782}$ and to model use of protective eyewear ${ }^{81-85}$ was more effective. Phone messages to remind workers to use protective eyewear also appeared to be an effective strategy to increase use. ${ }^{86}$

For mixed-ethnicity glaucoma patients, prerecorded translations were deemed equally effective as having translators available to enable visual field tests, ${ }^{40}$ and educational cartoons (designed to span language and cultural barriers) effectively improved adherence to amblyopia treatment. ${ }^{38} 39$

\section{Improving health}

Community low vision services improved self-reported functional vision (using a premeasure/postmeasure), ${ }^{87}$ and an educational programme for seasonal farm workers led to a reduction in self-reported eye pain. ${ }^{17}$ Measured, rather than self-reported, health outcomes were more difficult to improve with interventions, however, three preliminary studies aimed at reducing glycated haemoglobin (HbA1c) levels among people with diabetes showed encouraging results (one focused on building trust between patients and ophthalmologists, ${ }^{42}$ another on supportive interactions with community health workers $^{70}$ and another with an integrative web-based diabetes management system. ${ }^{88}$

Only one study included in this review reported incidence of blindness as an outcome. Baker $e t a l^{89}$ described a comprehensive, integrated programme (addressing several dimensions of access) which supported people with diabetes, living in low-income areas within Houston, USA (53\% Black, 30\% Latinx). In 1986, just prior to initiation of the programme, the incidence of blindness in this community was $9.5 / 1000$, but it reduced to $2.7 / 1000$ by 1989 as access to eyecare improved. Although the authors note that the confidence intervals do not indicate the change was statistically significant, clinically, they report the reduction in blindness to be very encouraging.

\section{What can we learn from authors' reflections on the potential to improve on interventions?}

Thematic analysis of the strengths, weaknesses and recommendations highlighted within each study resulted in several themes, the three most frequently noted are described briefly below.

\section{Relationship}

The most commonly noted factor attributed to the success of an intervention was a genuine relationship between participants and staff of the initiative. ${ }^{1742} 496165697282-8490-93$ For studies not collaborating with communities from the start, any social or culturally sensitive, aspects of the intervention tended to be highlighted as an important aspect of the project. ${ }^{38} 394452707194$ Although cultural concordance was an important factor, one study noted that rapport can be equally effective ${ }^{69}$ A related concept was that strategies effective in one community, or nondominant ethic group, were not always effective in another ${ }^{415664}$; communities are unique, and each local community should be involved in the development of interventions targeting them.

\section{Coordination}

Another commonly noted idea was that a coordinated, longitudinal approach was key to successful initiatives. ${ }^{435} 525461727689$ 95-104 Often this meant a branch of the project was dedicated to managing participants' transition from screening through to treatment and ongoing follow-up. It was noted by several studies that this type of approach required more resources than short, isolated interventions; both in terms of funding ${ }^{52} 5461$ and buy-in from many different professional staff. ${ }^{43} 102104$ A coordinated approach also meant being ready to treat a wide range of conditions. For example, prompt provision of refractive error and scheduling of cataract removal, as part of a diabetes intervention, was highlighted as more effective than having a limited, condition-specific scope. ${ }^{103}$

\section{Social gradient of target community}

Several authors noted that interventions to improve access to eyecare were limited by socioeconomic and cultural needs of target communities, ${ }^{36} 4357-59647991105$ 
suggesting the impact of underlying structural racism and generational poverty. The reliability of contact details appeared to be a common example of deeper socioeconomic issues ${ }^{36} 6491105$; those most at-risk of poor eye health were noted to have limited phone data, less stable housing and at times exhibit a reluctance to provide accurate identifying information (for fears related to immigration status, or being billed for help received) ${ }^{79}$ Poor literacy was thought to limit the impact of some interventions directly, ${ }^{58} 105$ and broader inequity across the social gradient (including education, employment and income) reflected the dimensions of access least addressed; the shortage of culturally concordant ophthalmologists was noted as a barrier to enhancing acceptability of services, ${ }^{43}$ and socioeconomic disadvantage limited patient's ability to pay for perceived or real costs, limiting access. ${ }^{57}$

\section{Grey literature}

The grey literature search identified 624 potential resources. Many of these highlighted the disproportionate burden of vision impairment among non-dominant ethnic minorities and the need for, planned, or newly established eyecare infrastructure to promote equity. Ultimately, we identified twelve resources that described initiatives to promote access directly, highlighted below (links in online supplemental file 1, table S1.3).

In the USA, the Affordable Care Act, the National Eye Institute, the American Academy of Ophthalmology, the National Association of Hispanic Nurses and some drug companies offer targeted education, and sometimes screening and treatment services (eg, EyeCare America, Travatan Project Focus, Partnership for Sight Initiative). Some local health boards (eg, in San Joaquin) and other initiatives (eg, Ventanillas de Salud and Juntos por la Salus) specifically target Latinx people living in the USA with health promotion programmes to promote access to healthcare, including eyecare. In Europe, initiatives in Glasgow and Wales appear more integrated, and a project in Finland focused on strengthening the provider side of access. The initiative in Finland was the only one we found which reported specific outcomes, notably that number of delayed follow-up eye appointments decreased dramatically after implementation.

Our grey literature search also highlighted more academic research from the USA underway, for example, the Screening and Intervention for Glaucoma and Eye Health through Telemedicine Programme.

\section{DISCUSSION}

\section{Summary of evidence}

What is the extent of the published literature?

Most studies were conducted in the USA (90\%), attempting to improve access for Black (48\%) and Latinx (29\%) populations. Issues with inequitable access to eyecare have been documented for high-income countries outside the USA (eg, in the UK, ${ }^{106}$ New Zealand ${ }^{107}$ and Sweden, ${ }^{108}$ ) yet, knowledge of the inequity and barriers does not appear to have translated to research about interventions to address them.

There were some similarities between this and our parallel review that synthesised eyecare service delivery models targeting Indigenous populations. ${ }^{6}$ Both reported a narrow geographic scope (USA: $90 \%$ in this review vs Australia: $67 \%^{6}$ ), both noted a focus on diabetic retinopathy $\left(45 \%\right.$ vs $\left.53 \%^{6}\right)$, and both noted that only about $30 \%$ of studies engaged communities in the design process. There were also some key differences; for example, glaucoma was targeted more for non-Indigenous, nondominant groups (18\%) than for Indigenous peoples $(2 \%){ }^{6}$ Interventions focused on trachoma appeared specific to Indigenous peoples in Australia ${ }^{6}$ (as this is not prevalent in other populations), whereas eye irritation/ injury experienced by seasonal farm workers was only highlighted for non-dominant, non-Indigenous populations in the USA. The existence of trachoma in Indigenous Australia and worker risk in the USA reflects the failure of high-income countries to meet basic environmental and social needs (including sanitation and workplace safety) of routinely marginalised people. That we identified some response to these disparities in the literature is encouraging, but more and better evidence is required to improve the eye health of these populations.

\section{What can we learn from reported effectiveness of interventions?}

Of the studies which reported at least one comparator, $40 \%$ used attendance at a follow-up eye exam after screening as the primary outcome measure. Interventions which improved attendance at follow-up tended to include personalised, supportive interactions between patients and providers, designed to help patients identify and address barriers to attendance. The approaches to improve access to eyecare highlighted here are similar to those summarised in other populations. A recent systematic review found tele-screening is likely cost-effective, especially in low-income areas. ${ }^{26} \mathrm{~A}$ systematic review of interventions to improve attendance for screening and follow-up for diabetic retinopathy also concluded that 'behavioural change techniques' (aimed at the patient), and quality improvements (aimed at the provider) were effective at improving attendance. They found that on average attendance increased by $12 \%$ compared with no intervention, but noted that communities with lower baseline levels of attendance benefited the most from intervention. ${ }^{27}$ Indeed, several studies highlighted in this review showed improvements over 20\%. ${ }^{52616668}$

It is important to note that focusing on behavioural change can decrease health outcomes, if it is at the cost of considering the complex social gradient in which non-dominant communities exist. ${ }^{109}$ Several larger initiatives captured in this review appeared to appreciate the risks of putting the onus on already marginalised patients. These studies tended to weave elements of behavioural activation within more comprehensive services, including convenient community screening, streamlining referral and treatment services including 
prompt booking of required appointments in convenient locations, culturally concordant staff and financial incentives. Although there is limited information about health outcomes captured in this review, one encouraging study in the USA, targeting urban, low-income, mostly Black patients with diabetes demonstrated a decrease in the incidence of blindness as a result of an intervention targeting several aspects of access in a coordinated manner. ${ }^{89}$

\section{What can we learn from authors' reflections on the potential to} improve on interventions?

Authors' reflections emphasised the need for enabling relationships between provider and patient, coordination of steps from screening through to treatment and the need to address wider issues of poverty and inequity in health and education. The first two can be addressed through improved interventions, but the latter requires wider systemic change. Programmes to enhance ethnic diversity in ophthalmology programmes ${ }^{110}$ is an example of how institution-level changes could improve currently hard to address aspects of access. However, these programmes will take time to have an impact, as will the further steps of addressing poor representation in ophthalmology leadership, ${ }^{111}$ and structural inequity more generally.

\section{Considering all three questions together}

Despite authors noting that genuine relationships between patients and providers is a key element to success, only $30 \%$ of the studies involved the target community as part of the design process. Similarly, despite author reflections on the importance of consistent, longitudinal initiatives, most published projects were of limited scope and duration. Studies which achieved both these targets were generally housed within ongoing highly collaborative initiatives, delivering interventions which prioritised empowering patients while providing better services in a coordinated way. However, studies within these larger initiatives were less likely to report an effective study outcome than more isolated studies (despite larger studies being evaluated at a slightly higher rate). This appeared to be because the larger initiatives took more flexible, longer-term approaches, tended to target the most hard-to-reach communities, and focused on more meaningful outcomes (eg, attendance at follow-up eye exam rather than knowledge on a survey), which are harder to achieve. Taken together, appropriate measures of impact are an important area of future research, perhaps frameworks such as reach, efficacy, adoption, implementation and maintenance ${ }^{96}$ start to address this issue. There are additional unpublished initiatives which provide education, screening and treatment to promote equitable access to eyecare, initiated from within various sectors, but few appear to be resourcing at-risk communities to provide solutions, or highlighting communitydriven initiatives.

\section{Study limitations}

Several definitions or concepts were simplified to facilitate a usable summary, and some categorisations were subjective. In particular, the population of interest (as stated in the Methods section, and the protocoll ${ }^{28}$ ) is difficult to define. Future work may benefit from broadening the target population to look at those with low socioeconomic status in high-income countries or, alternatively, narrowing the target population to look at a specific non-dominant ethnic group in a specific place. The results of our review suggest the bulk of existing research is focused on Black people living in low socioeconomic areas of USA, which may not generalise to other communities in other high-income countries (with different politics, experience of structural racism and eyecare systems).

Many studies noted reliance on church groups, notfor-profit support networks, or community boards to develop, guide and support the initiatives described in publications. Collaboration with these community programmes (often setup to support specific, but not necessarily ethnicity-defined groups, such as recent immigrants, homeless populations or those living in poverty) appeared integral for successful interventions. These types of initiatives should be further explored, as there are likely to be many which are unpublished. Even the grey literature did not capture grassroots initiatives, rather, relevant resources appeared to be press releases from larger associations.

\section{CONCLUSION}

In this scoping review, we have mapped published interventions aimed at improving access to eyecare for nondominant ethnic groups. Most interventions were carried out in the USA, targeting Black and Latinx communities, and described screening programmes targeting people with diabetic retinopathy and glaucoma. More research is needed to address the inequities in access to eyecare in high-income countries beyond the USA. Only $30 \%$ of studies included the target population in planning and implementation, the majority of which were larger, coordinated initiatives. Most evaluated interventions focused on improving attendance at a follow-up eye exam after screening; among these, personalised support to help patients address barriers to attendance appeared most effective. However, placing onus on patient behavioural change has limits, and several papers suggested that inequity in eyecare accessibility will persist if structural racism and generational poverty are not overcome. While striving to address underlying socioeconomic and cultural needs, this review suggests that building genuine relationships between patients and providers (including collaborative intervention design) and establishing longitudinal coordinated initiatives (which address many barriers to access simultaneously) are important starting points. 


\section{Dissemination statement}

The scoping review outlined here is part of a larger study to improve access to eyecare services for Indigenous and non-Indigenous ethnic groups in Aotearoa/New Zealand. The findings will be useful to policymakers, health service managers and clinicians responsible for eyecare services in New Zealand, as well as in other countries with similar marginalised population groups. We will develop an accessible summary of the results for posting on institutional websites and dissemination at stakeholder meetings.

\section{Author affiliations}

${ }^{1}$ School of Optometry \& Vision Science, Faculty of Medical and Health Sciences, The University of Auckland, Auckland, New Zealand

${ }^{2}$ Centre for Health Equity Training Research \& Evaluation, University of New South Wales, Sydney, New South Wales, Australia

${ }^{3}$ Centre for Primary Health Care and Equity, University of New South Wales, Sydney, New South Wales, Australia

${ }^{4}$ Ingham Institute for Applied Medical Research, Liverpool, New South Wales, Australia

${ }^{5}$ School of Social Sciences, University of New South Wales, Sydney, New South Wales, Australia

${ }^{6}$ International Centre for Eye Health, London School of Hygiene \& Tropical Medicine, London, UK

${ }^{7}$ School of Population Health, Faculty of Medical and Health Sciences, The University of Auckland, Auckland, New Zealand

${ }^{8}$ Performance Improvement, Auckland District Health Board, Auckland, New Zealand

${ }^{9}$ Moorfields Eye Hospital, London, UK

Contributors JR conceived the idea for the review. LMH, JB, AY, HB, CG, RP-J and JR did the screening and data extraction. LMH and JR drafted and revised the manuscript with analytical help and suggestions from JB, AY, HB, CG, MH, RP-J, MJB and JRE.

Funding This work was supported by The University of Auckland Faculty Research Development Fund grant number 3716758 . JR's position at the University of Auckland is funded by the Buchanan Charitable Foundation, New Zealand. MJB is supported by the Wellcome Trust (207472/Z/17/Z). LMH is a Rutherford Postdoctoral Research Fellow, funded by the Royal Society of New Zealand (3720000) and School of Medicine Foundation (3722073), CG holds a National Heart Foundation Fellowship.

Competing interests None declared.

Patient consent for publication Not required.

Provenance and peer review Not commissioned; externally peer reviewed.

Data availability statement All data relevant to the study are included in the article or uploaded as online supplemental information.

Supplemental material This content has been supplied by the author(s). It has not been vetted by BMJ Publishing Group Limited (BMJ) and may not have been peer-reviewed. Any opinions or recommendations discussed are solely those of the author(s) and are not endorsed by BMJ. BMJ disclaims all liability and responsibility arising from any reliance placed on the content. Where the content includes any translated material, BMJ does not warrant the accuracy and reliability of the translations (including but not limited to local regulations, clinical guidelines, terminology, drug names and drug dosages), and is not responsible for any error and/or omissions arising from translation and adaptation or otherwise.

Open access This is an open access article distributed in accordance with the Creative Commons Attribution 4.0 Unported (CC BY 4.0) license, which permits others to copy, redistribute, remix, transform and build upon this work for any purpose, provided the original work is properly cited, a link to the licence is given, and indication of whether changes were made. See: https://creativecommons.org/ licenses/by/4.0/.

\section{ORCID iDs}

Lisa M Hamm http://orcid.org/0000-0003-2777-7146

Aryati Yashadhana http://orcid.org/0000-0003-2573-8637
Helen Burn http://orcid.org/0000-0002-1469-8169

Joanna Black http://orcid.org/0000-0002-5100-8796

Corina Grey http://orcid.org/0000-0003-1751-1934

Matire Harwood http://orcid.org/0000-0003-1240-5139

Roshini Peiris-John http://orcid.org/0000-0001-7812-2268

Matthew J Burton http://orcid.org/0000-0003-1872-9169

Jennifer R Evans http://orcid.org/0000-0002-6137-2030

Jacqueline Ramke http://orcid.org/0000-0002-5764-1306

\section{REFERENCES}

1 Tranforming our world: the 2030 agenda for sustainable development: United Nations 2015

2 Indigenous peoples and ethnic minorities: marginalization is the norm. promoting inclusion through social protection United Nations 2018.

3 Holmes L, Enwere M, Williams J. Black-White risk differentials in COVID-19 (SARS-COV2) transmission, mortality and case fatality in the United States: translational epidemiologic perspective and challenges. International Journal of Environmental Research and Public Health 2020;17:4322.

4 Bailey ZD, Krieger N, Agénor M, et al. Structural racism and health inequities in the USA: evidence and interventions. Lancet 2017;389:1453-63.

5 Del Pino S, Sánchez-Montoya SB, Guzmán JM. Health inequalities amongst people of African descent in the Americas, 2005-2017: a systematic review of the literature. International Journal of Environmental Research and Public Health 2019;16:3302.

6 Burn $\mathrm{H}$, Hamm L, Black J, et al. Eye care delivery models to improve access to eye care for Indigenous peoples in high-income countries: a scoping review. BMJ Glob Health 2021;6:e004484.

7 Islam NS, Kwon SC, Wyatt LC, et al. Disparities in diabetes management in Asian Americans in New York City compared with other racial/ethnic minority groups. Am J Public Health 2015;105 Suppl 3:S443-6.

8 Varadaraj V, Frick KD, Saaddine JB. Trends in eye care use and eyeglasses affordability: the US National health interview survey, 2008-2016Trends in eye care use and eyeglasses AffordabilityTrends in eye care use and eyeglasses affordability. JAMA Ophthalmology 2019;137:391-8.

9 Fang CY, Ragin CC. Addressing disparities in cancer screening among U.S. immigrants: progress and opportunities. Cancer Prev Res 2020;13:253-60.

10 Tabb KM, Larrison CR, Choi S, et al. Disparities in health services use among Multiracial American young adults. J Immigr Minor Health 2016;18:1462-9.

11 D'Angelo D, Di Nitto M, Giannarelli D, et al. Inequity in palliative care service full utilisation among patients with advanced cancer: a retrospective cohort study. Acta Oncol 2020;59:620-7.

12 Baker RS, Bazargan M, Bazargan-Hejazi S, et al. Access to vision care in an urban low-income multiethnic population. Ophthalmic Epidemiol 2005;12:1-12.

13 Varma R, Mohanty SA, Deneen J, et al. Burden and predictors of undetected eye disease in Mexican-Americans: the Los Angeles Latino eye study. Med Care 2008;46:497-506.

14 Al-Aswad LA, Joiner DB, Wang X. Screening for glaucoma in populations at high risk: the eye screening New York project. Cogent Medicine 2017;4:1367059.

15 Martin SA, Frutiger EA. Vision stations: addressing corrective vision needs with low-cost technologies. Glob Adv Health Med 2015;4:46-51.

16 Zhang X. Eye care in the United States. Archives of Ophthalmology 2007;125:411.

17 Earle-Richardson G, Wyckoff L, Carrasquillo M, et al. Evaluation of a community-based participatory farmworker eye health intervention in the "black dirt" region of New York state. Am J Ind Med 2014:57:1053-63.

18 Heslin KC, Casey R, Shaheen MA, et al. Racial and ethnic differences in unmet need for vision care among children with special health care needs. Arch Ophthalmol 2006;124:895.

19 Baker H, Murdoch IE. Can a public health intervention improve awareness and health-seeking behaviour for glaucoma? $\mathrm{Br} \mathrm{J}$ Ophthalmol 2008;92:1671-5.

20 Levesque J-F, Harris MF, Russell G. Patient-Centred access to health care: conceptualising access at the interface of health systems and populations. Int J Equity Health 2013;12:18.

21 Hall AG. Medicaid's impact on access to and utilization of health care services among racial and ethnic minority children. J Urban Health 1998;75:677-92. 
22 Rutledge EO. The struggle for equality in healthcare continues. $J$ Healthc Manag 2001;46:313-26.

23 Wilson D, Heaslip V, Jackson D. Improving equity and cultural responsiveness with marginalised communities: understanding competing worldviews. J Clin Nurs 2018;27:3810-9.

24 Cicinelli MV, Marmamula S, Khanna RC. Comprehensive eye care - Issues, challenges, and way forward. Indian J Ophthalmol 2020;68:316-23.

25 Ramke J, Petkovic J, Welch V. Interventions to improve access to cataract surgical services and their impact on equity in lowand middle-income countries. Cochrane Database of Systematic Reviews 2017:11.

26 Avidor D, Loewenstein A, Waisbourd M, et al. Cost-Effectiveness of diabetic retinopathy screening programs using telemedicine: a systematic review. Cost Eff Resour Alloc 2020;18:16.

27 Lawrenson JG, Graham-Rowe E, Lorencatto F, et al. Interventions to increase attendance for diabetic retinopathy screening. Cochrane Database Syst Rev 2018;2018.

28 Hamm LM, Black J, Burn $\mathrm{H}$, et al. Interventions to promote access to eye care for non-Indigenous, non-dominant ethnic groups in high-income countries: a scoping review protocol. BMJ Open 2020;10:e033775.

29 Tricco AC, Lillie E, Zarin W, et al. PRISMA extension for scoping reviews (PRISMA-ScR): checklist and explanation. Ann Intern Med 2018;169:467-73.

30 Merino ME, Tileagă $\mathrm{C}$. The construction of ethnic minority identity: a discursive psychological approach to ethnic self-definition in action. Discourse and Society 2011;22:86-101.

31 Ford CL, Harawa NT. A new conceptualization of ethnicity for social epidemiologic and health equity research. Soc Sci Med 2010;71:251-8.

32 Benson C. School access for children from nondominant ethnic and linguistic communities fixing the broken promise of education for all: findings from the global initiative on out-of-school children UNICEF 2014.

33 Burn $\mathrm{H}$, Black J, Harwood M, et al. Eye care delivery models to improve access to eye care for Indigenous people in highincome countries: protocol for a scoping review. BMJ Open 2019;9:e029214.

34 Watson T, Orel-Bixler D, Haegerstrom-Portnoy G. Early visualevoked potential acuity and future behavioral acuity in cortical visual impairment. Optom Vis Sci 2010;87:80-6.

35 Glaser BG, Strauss AL. The discovery of grounded theory: strategies for qualitative research. London: Routledge, 2009.

36 Bush K, Thomas R, Raymond NT, et al. Cluster randomised controlled trial evaluation of a link Worker-delivered intervention to improve uptake of diabetic retinopathy screening in a South Asian population. Diab Vasc Dis Res 2014;11:294-7.

37 Feldman F, Lee M. An eye screening program in a ChineseCanadian community. Can J Ophthalmol 1977;12:121-3.

38 Tjiam AM, Holtslag G, Van Minderhout HM. Randomised comparison of three tools for improving compliance with occlusion therapy: an educational Cartoon story, a reward calendar, and an information leaflet for parents. Graefes Archive for Clinical \& Experimental Ophthalmology 2013;251:321-9.

39 Tjiam AM, Holtslag G, Vukovic E, et al. An educational Cartoon accelerates amblyopia therapy and improves compliance, especially among children of immigrants. Ophthalmology 2012;119:2393-401.

40 Nesher R, Ever-Hadani P, Epstein E, et al. Overcoming the language barrier in visual field testing. J Glaucoma 2001;10:203-5.

41 Lee SJ, McCarty CA, Sicari C, et al. Recruitment methods for community-based screening for diabetic retinopathy. Ophthalmic Epidemiol 2000;7:209-18.

42 Rovner BW, Casten RJ. Trust and glycemic control in black patients with diabetic retinopathy: a pilot study. Diabetes Spectr 2019;32:152-5.

43 Zhao D, Guallar E, Bowie JV, et al. Improving follow-up and reducing barriers for eye screenings in communities: the stop glaucoma study. Am J Ophthalmol 2018:188:19-28.

44 Rhodes LA, Huisingh CE, McGwin G, et al. Eye care quality and accessibility improvement in the community (equality): impact of an eye health education program on patient knowledge about glaucoma and attitudes about eye care. Patient Relat Outcome Meas 2016:7:37-48.

45 Moussa M, Sherrod D, Choi J. An e-health intervention for increasing diabetes knowledge in African Americans. Int J Nurs Pract 2013;19 Suppl 3:36-43.

46 Navuluri RB. Diabetic retinopathy screening among Hispanics in LEA County, new Mexico. J Health Care Poor Underserved 2000;11:430-43.
47 Wagner H, Pizzimenti JJ, Daniel K, et al. Eye on diabetes: a multidisciplinary patient education intervention. Diabetes Educ 2008;34:84-9.

48 Owsley C, McGwin G, Stalvey BT, et al. Educating older African Americans about the preventive importance of routine comprehensive eye care. J Natl Med Assoc 2008;100:1089-95.

49 Frazier M, De La Cruz N, Garcés IC. Development of educational materials to improve rates of early eye care for Hispanic children. $J$ Immigr Minor Health 2012;14:608-16.

50 Kharod BV, Johnson PB, Nesti HA, et al. Effect of written instructions on accuracy of self-reporting medication regimen in glaucoma patients. J Glaucoma 2006;15:244-7.

51 Hark L, Waisbourd M, Myers JS, et al. Improving access to eye care among persons at high-risk of glaucoma in philadelphiadesign and methodology: the Philadelphia glaucoma detection and treatment project. Ophthalmic Epidemiol 2016;23:122-30.

52 Davis RM, Fowler S, Bellis K, et al. Telemedicine improves eye examination rates in individuals with diabetes: a model for eye-care delivery in underserved communities. Diabetes Care 2003;26:2476.

53 Jani PD, Forbes L, Choudhury A, et al. Evaluation of diabetic retinal screening and factors for ophthalmology referral in a telemedicine network. JAMA Ophthalmol 2017;135:706-14.

54 Olayiwola JN, Sobieraj DM, Kulowski K, et al. Improving diabetic retinopathy screening through a statewide telemedicine program at a large federally qualified health center. $J$ Health Care Poor Underserved 2011;22:804-16.

55 Taylor CR, Merin LM, Salunga AM, et al. Improving diabetic retinopathy screening ratios using telemedicine-based digital retinal imaging technology: the Vine Hill study. Diabetes Care 2007;30:574-8.

56 Mehranbod C, Genter P, Serpas L. Automated reminders improve retinal screening rates in low income, minority patients with diabetes and correct the African American disparity. Journal of Medical Systems 2020;44:17.

57 Owsley C, McGwin G, Searcey K, et al. Effect of an eye health education program on older African Americans' eye care utilization and attitudes about eye care. J Natl Med Assoc 2013;105:69-76.

58 Ellish NJ, Royak-Schaler R, Higginbotham EJ. Tailored and targeted interventions to encourage dilated fundus examinations in older African Americans. Arch Ophthalmol 2011;129:1592-8.

59 Zhang X, Williams DE, Beckles GL, et al. Diabetic retinopathy, dilated eye examination, and eye care education among African Americans, 1997 and 2004. J Natl Med Assoc 2009;101:1015-21.

60 Aleo CL, Murchison AP, Dai Y, et al. Improving eye care followup adherence in diabetic patients with ocular abnormalities: the effectiveness of patient contracts in a free, pharmacy-based eye screening. Public Health 2015;129:996-9.

61 Anderson RM, Musch DC, Nwankwo RB, et al. For the patient. eye screening can prevent eye disease. personalized follow-up increases return rate at urban eye disease screening clinics for African Americans with diabetes: results of a randomized trial. Ethn Dis 2003;13:149.

62 Pizzi LT, Zangalli CS, Murchison AP, et al. Prospective randomized controlled trial comparing the outcomes and costs of two eyecare adherence interventions in diabetes patients. Appl Health Econ Health Policy 2015;13:253-63.

63 Prezio EA, Balasubramanian BA, Shuval K, et al. Evaluation of quality improvement performance in the community diabetes education (code) program for uninsured Mexican Americans: results of a randomized controlled trial. Am J Med Qual 2014;29:124-34.

64 Meng Y-Y, Diamant A, Jones J, et al. Racial and ethnic disparities in diabetes care and impact of Vendor-Based disease management programs. Diabetes Care 2016;39:743-9.

65 Casten RJ, Brawer R, Haller JA. Trial of a behavioral intervention to increase dilated fundus examinations in African-Americans aged over 65 years with diabetes. Expert Review of Ophthalmology 2011;6:593-601.

66 Weiss DM, Casten RJ, Leiby BE, et al. Effect of behavioral intervention on dilated fundus examination rates in older African American individuals with diabetes mellitus: a randomized clinical trial. JAMA Ophthalmol 2015;133:1005-12.

67 Winters D, Casten R, Rovner B, et al. Cost-Effectiveness of behavior activation versus supportive therapy on adherence to eye exams in older African Americans with diabetes. Am J Med Qual 2017;32:661-7.

68 Basch CE, Walker EA, Howard CJ, et al. The effect of health education on the rate of ophthalmic examinations among African Americans with diabetes mellitus. Am J Public Health 1999;89:1878-82.

69 Jones HL, Walker EA, Schechter CB, et al. Vision is precious: a successful behavioral intervention to increase the rate of screening 
for diabetic retinopathy for inner-city adults. Diabetes Educ 2010;36:118-26.

70 Vaughan EM, Johnston CA, Cardenas VJ. Integrating CHWs as part of the team leading diabetes group visits: a randomized controlled feasibility study. Diabetes Educator 2017;43:589-99.

71 Walker EA, Schechter CB, Caban A, et al. Telephone intervention to promote diabetic retinopathy screening among the urban poor. Am $J$ Prev Med 2008;34:185-91.

72 Hark LA, Johnson DM, Berardi G, et al. A randomized, controlled trial to test the effectiveness of a glaucoma patient navigator to improve appointment adherence. Patient Prefer Adherence 2016;10:1739-48.

73 Hark L, Acito M, Adeghate J, et al. Philadelphia telemedicine glaucoma detection and follow-up study: ocular findings at two health centers. J Health Care Poor Underserved 2018;29:1400-15.

74 Hark LA, Adeghate J, Katz LJ, et al. Philadelphia telemedicine glaucoma detection and follow-up study: cataract classifications following eye screening. Telemed J E Health 2020;26:992-1000.

75 Hark LA, Myers JS, Ines A, et al. Philadelphia telemedicine glaucoma detection and follow-up study: confirmation between eye screening and comprehensive eye examination diagnoses. $\mathrm{Br} \mathrm{J}$ Ophthalmol 2019;103:1820-6.

76 Rodriguez E, Srivastava A, Landau M. Increasing screening followup for vulnerable children: a partnership with school nurses. Int $J$ Environ Res Public Health 2018;15:25.

77 Hong K, Dragan K, Glied S. Seeing and hearing: the impacts of new York City's universal pre-kindergarten program on the health of low-income children. J Health Econ 2019;64:93-107.

78 van Zyl T, Su Z, Zhou E, et al. Providing prescheduled appointments as a strategy for improving follow-up compliance after community-based glaucoma screening: results from an urban underserved population. $J$ Community Health 2015;40:27-33.

79 Chedid EH, Golden QR, Jager RD. Operational challenges in delivery of a charity care program for diabetic retinopathy screening in an urban setting. Permanente Journal 2013;17:21-5.

80 Jenkins C, McNary S, Carlson BA, et al. Reducing disparities for African Americans with diabetes: progress made by the reach 2010 Charleston and Georgetown diabetes coalition. Public Health Rep 2004;119:322-30.

81 Forst L, Lacey S, Chen HY, et al. Effectiveness of community health workers for promoting use of safety eyewear by Latino farm workers. Am J Ind Med 2004;46:607-13.

82 Tovar-Aguilar JA, Monaghan PF, Bryant CA, et al. Improving eye safety in citrus harvest crews through the acceptance of persona protective equipment, community-based participatory research, social marketing, and community health workers. J Agromedicine 2014:19:107-16.

83 Luque JS, Monaghan P, Contreras RB, et al. Implementation evaluation of a culturally competent eye injury prevention program for citrus workers in a Florida migrant community. Prog Community Health Partnersh 2007;1:359-69.

84 Monaghan PF, Bryant CA, McDermott RJ, et al. Adoption of safety eyewear among citrus harvesters in rural Florida. $J$ Immigr Minor Health 2012;14:460-6.

85 Monaghan PF, Forst LS, Tovar-Aguilar JA, et al. Preventing eye injuries among citrus harvesters: the community health worker model. Am J Public Health 2011;101:2269-74.

86 Snipes SA, Smyth JM, Murphy D, et al. Provision increases reported PPE use for Mexican immigrant Farmworkers: an mHealth pilot study. J Occup Environ Med 2015;57:1343-6.

87 Draper EM, Feng R, Appel SD, et al. Low vision rehabilitation for adult African Americans in two settings. Optom Vis Sci 2016;93:673-82.

88 Welch G, Allen NA, Zagarins SE. Comprehensive diabetes management program for poorly controlled Hispanic type 2 patients at a community health center. Diabetes Educator 2011;37:680-8.

89 Baker SB, Vallbona C, Pavlik V, et al. A diabetes control program in a public health care setting. Public Health Rep 1993;108:595-605.

90 Anderson RM, Wolf FM, Musch DC, et al. Conducting communitybased, culturally specific, eye disease screening clinics for urban African Americans with diabetes. Ethn Dis 2002;12:404-10.
91 DeNomie M, Medić V, Castro A, et al. Lessons learned from a community-academic project using telemedicine for eye screening among urban Latinos. Prog Community Health Partnersh 2019;13:183-9.

92 Sanspree MJ, Allison C, Goldblatt SH, et al. Alabama Black Belt eye care-optometry giving back. Optometry 2008;79:724-9.

93 Dreer LE, Owsley C, Campbell L, et al. Feasibility, patient acceptability, and preliminary efficacy of a culturally informed, health promotion program to improve glaucoma medication adherence among African Americans: "glaucoma management optimism for african americans living with glaucoma" (GOAL). Curr Eye Res 2016;41:50-8.

94 Shahid K, Kolomeyer AM, Nayak NV, et al. Ocular telehealth screenings in an urban community. Telemed $J$ E Health 2012:18:95-100.

95 Winters JE, Messner LV, Gable EM, et al. Coordinating eye and primary medical care in a low-income and uninsured population: the experience of the vision of hope health alliance. Optometry 2008;79:730-6.

96 Sapru S, Berktold J, Crews JE, et al. Applying RE-AIM to evaluate two community-based programs designed to improve access to eye care for those at high-risk for glaucoma. Eval Program Plann 2017;65:40-6.

97 Waisbourd M, Pruzan NL, Johnson D, et al. The Philadelphia glaucoma detection and treatment project: detection rates and initial management. Ophthalmology 2016;123:1667-74.

98 Adeghate JO, Hark LA, Brown H, et al. Philadelphia glaucoma detection and treatment project: ocular outcomes and adherence to follow-up at a single health centre. Can J Ophthalmol 2019;54:717-22.

99 Miller PG. Vision screening for migrant children. Child Today 1976;5:6-7.

100 Mehravaran S, Duarte PB, Brown SI, et al. The UCLA preschool vision program, 2012-2013. J Aapos 2016:20:63-7.

101 Preslan MW, Novak A. Baltimore vision screening project. Ophthalmology 1996;103:105-9.

102 Kattouf VM, Scharre J, McMahon J, et al. Comprehensive vision care in urban communities: the pediatric outreach program. Optometry 2009;80:29-35.

103 Owsley C, McGwin G, Lee DJ, et al. Diabetes eye screening in urban settings serving minority populations: detection of diabetic retinopathy and other ocular findings using telemedicine. JAMA Ophthalmol 2015;133:174-81.

104 Zhao D, Guallar E, Gajwani P, et al. Optimizing glaucoma screening in high-risk population: design and 1-year findings of the screening to prevent (stop) glaucoma study. Am J Ophthalmol 2017;180:18-28.

105 Zheng CX, Hu WD, Tran J, et al. Barriers to receiving follow-up eye care and detection of Non-Glaucomatous ocular pathology in the Philadelphia glaucoma detection and treatment project. $J$ Community Health 2016;41:359-67.

106 Kliner M, Fell G, Gibbons C, et al. Diabetic retinopathy equity profile in a multi-ethnic, deprived population in northern England. Eye 2012;26:671-7.

107 Ramke J, Jordan V, Vincent AL, et al. Diabetic eye disease and screening attendance by ethnicity in New Zealand: a systematic review. Clin Exp Ophthalmol 2019;47:937-47.

108 Smirthwaite G, Lundström M, Wijma B. Inequity in waiting for cataract surgery - an analysis of data from the Swedish National Cataract Register. International Journal for Equity in Health 2016;15.

109 Centre for Health Equity Training, Research and Evaluation (CHETRE). Behaviour change: its (per)verse relation with health equity. The briefing equity evidence report. Sydney: Centre for Health Equity Training, Research and Evaluation (CHETRE), 2021.

110 Olivier MMG, Forster S, Carter KD, et al. Lighting a pathway: the minority ophthalmology mentoring program. Ophthalmology 2020;127:848-51

111 Yashadhana A, Clarke NA, Zhang JH, et al. Gender and ethnic diversity in global ophthalmology and optometry association leadership: a time for change. Ophthalmic Physiol Opt 2021;41:623-9. 